\title{
Mathematical Modelling: A Study of Corruption in Various fields of the Society
}

\author{
Sayaji Rastum Waykar \\ Research Scholar, JJT University, Rajasthan, C/O Assistant Professor, Department of Mathematics \\ Yashwantrao Chavan Mahavidyalaya, Halkarni Tal: Chandgad, Dist: Kolhapur, Maharashtra (India).
}

\begin{abstract}
In this paper we have to study on the problem of 'Corruption' in different ways by using mathematical modelling. The problem of corruption is everywhere, so we will try to find the solution for the problem of 'removing corruption' in the society. Therefore, how to measure the corruption in the society of any field or any country in the world? So, we have found the formula that is Mathematical corruption model for measuring the corruption in the society of any field or any country of the world. When we measure the corruption in the society then there will be no difficult to remove the corruption from the society of any country in the world. So we have to take some illustrations for measuring the corruption in various fields of the country India.
\end{abstract}

Keywords: mathematical thinking, corruption mentality, modelling, applied.

\section{Introduction:}

We have to study some formulae on "Corruption" in the difference equation form is known as Mathematical Corruption Model. The objective of mathematical analysis is to provide some general understanding of discrete models for corruption such as this, so that we can determine how the system behaves without having to iterate it numerically. Numerical iteration tells us only what one particular trajectory of the system will be through time. We had potentially had to do much iteration to get a general picture of the behavior of the system, and mathematical analysis saves us from having to do this. These mathematical discrete models for corruptions formulae are as of the following:

- Mathematical Discrete Model of Corruption with the Difference Equation Formula:

$$
\mathrm{C}=C_{0}(K+1)^{t}
$$

- Mathematical Decay of Discrete Model of Corruption (or MCC Model) with the Difference Equation Formula:

$$
\mathrm{C}=C_{0}(K+1)^{-t}
$$

- Mathematical Corruption-Development Model with the Difference Equation Formula:

$$
\mathrm{D}(\mathrm{C})=\mathrm{D}(\mathbf{0})[\mathbf{1}+\mathrm{K}]^{\mathrm{C}}
$$

- Mathematical Development Model with the Difference Equation Formula:

$$
\mathrm{D}=D_{0}[1+K]^{\mathrm{t}}
$$

- Mathematical E-virus Constant Model with Related Time in the Difference Equation Formula:

$$
\mathbf{K}=\left[\frac{C(t)}{C(0)}\right]^{\frac{1}{t}}-1,-1<\mathbf{K}<\mathbf{1}
$$

- Mathematical E-virus Constant Model with Related Corruption in the Difference Equation Formula:

$$
\mathbf{K}=\left[\frac{D(C)}{D(0)}\right] \frac{\mathbf{1}}{\bar{c}}-\mathbf{1},-1<\mathbf{K}<\mathbf{1}
$$

Note that if the value of $\mathbf{K}$ is more than 1 then we choose or take the value of $\mathbf{K}$ is approximately to 1 but not equal to 1.

All the above Mathematical discrete corruption model formulae are used for measuring as well as controlling the corruption in the society of any country of the world.

\section{Methodology:}

We have to use the seven steps of mathematical modelling process for solving the problem of corruption in the society of any country of the world. The mathematical modelling process is the translation from rest of the world to the Mathematics world. Also we know that rest of world means outside mathematics that is nature, society, everyday life and other scientific disciplines. So first we understand the real problem 'corruption' then make a real model after that by using mathematical operations we get mathematical model then using some assumptions we get mathematical result then interpreting this mathematical result if it is true we get the final result otherwise revise the model. This process is known as 'Mathematical modeling'. It is as follows: 


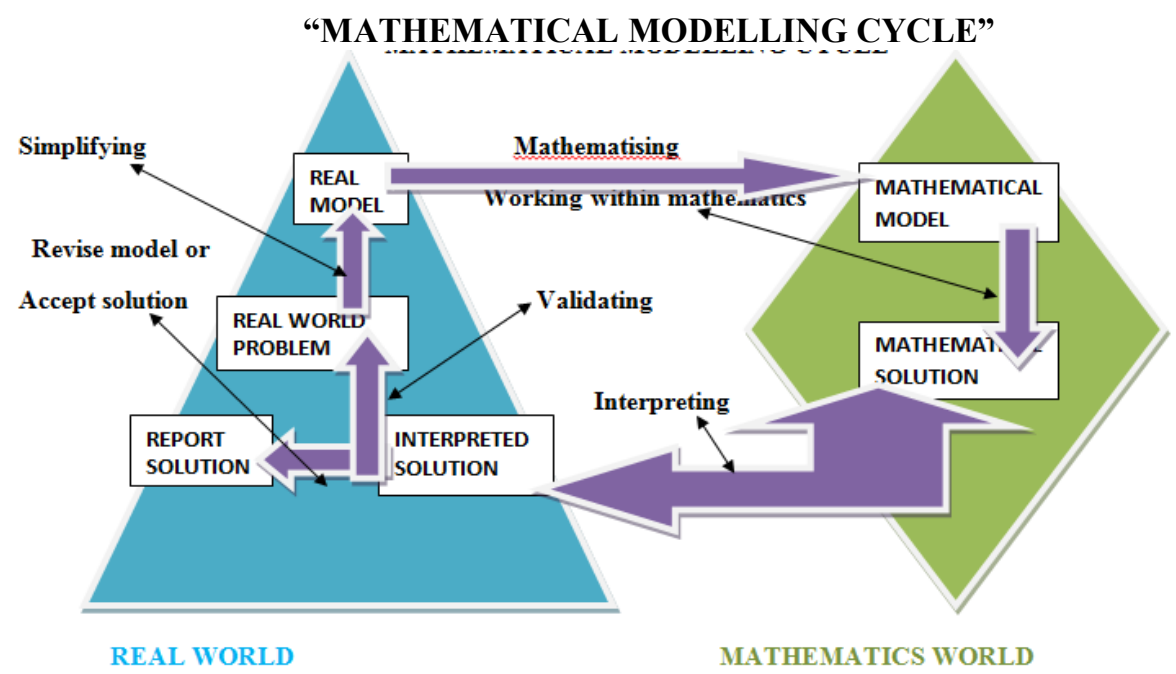

Fig.1: The "Answer Plan" for Mathematical modeling process.

Mathematical modelling means "Translation from real world problems into Mathematics world."

III. Some Illustrations for measuring the Corruption in the Society:

Corruption may be defined as an illegal selling of a public entity that is government, public corporation, public charity etc. property or services for gain or bribe to the seller not to the public entity. In the society Corruption problems are everywhere in various cities and various villages in any country of the world. We have observed that the 'Common Man' in the society faced Corruption problem in obtaining 11 (eleven) different public services such as:

* Income Tax bureaucracy * Municipal Services * Judiciary * Rural Financial Institution (RFI) * Land Administration * Police (crime/traffic) * Education: Public Schools/colleges and other Institutions * Water Suppliers * Electricity Suppliers * Government Hospitals * Ration/Aadhar Card Suppliers. Here we have to explain one illustration for public services. There is as of the following:

\section{1: Income Tax bureaucracy:}

We have known that in India the most of the people have paid Income tax from 15 August, 1947 but also among these maximum numbers of people have not paid (full or less) or miss the Income tax. So I will try to find how many people's have paid Income tax or not or less from 15 August 1947.

We assume that first ten years period that is 15 August, 1947 to 15 August, 1957; the 0.5\% people of total population 35 crore had paid Income tax. That is $C(0)=0.175$ crore when $t=0$; after $t=10$ years Mathematical Model Period, $\mathrm{C}(\mathrm{t})=0.30625$ crore that is $7 / 4$ of $\mathrm{C}(0)$. Therefore we find the value of Mathematical E- virus Constant $\mathbf{K}$.

We know that the Mathematical E-virus Constant Model with related time formula in the difference equation form,

$$
\begin{aligned}
& \therefore & K & =\left[\frac{C(t)}{C(0)}\right]^{\frac{1}{t}}-1 \\
& \therefore & 1+K & =\left[\frac{0.30625}{0.175}\right]^{\frac{1}{10}}
\end{aligned}
$$

Putting this value in the following Mathematical corruption model with difference equation form, we get

$$
\begin{array}{ll}
\therefore & \mathrm{C}=C_{0}[1+\mathrm{K}]^{t} \\
\therefore & \mathrm{C}=0.175 \times\left[\frac{0.30625}{\mathbf{0 . 1 7 5}}\right]^{\frac{t}{10}} \\
\therefore & \mathrm{C}=\mathbf{0 . 1 7 5} \times[\mathbf{1 . 7 5}]^{\frac{t}{10}}
\end{array}
$$

This is known as Mathematical Model base Corruption with related time formula. The following table shows that the total number of Income tax paid holder or not (full or less) paid holder with related Mathematical model period $\mathrm{t}=10$ years from 15 August, 1947 to 15 August, 2001 and $\mathrm{t}=1$ year from 15 August 2001 to 15 August, 2014. It is as,

When $\mathrm{t}=10$ years from base; that is 15 August, 1957, what is $\mathrm{C}=$ ?

$$
\begin{array}{lll}
\text { From }(\mathrm{I}), & \therefore & \mathrm{C}=0.175 \times[1.75]^{\frac{10}{10}} \\
& \therefore & \mathrm{C}=0.175 \times[1.75]^{\frac{1}{1}}
\end{array}
$$




$$
\begin{array}{rrr}
\therefore \quad & C=0.175 \times 1.75 \\
& & C=0.30625 \text { crore }
\end{array}
$$

When $\mathrm{t}=20$ years from base; that is 15 August, 1967, what is $\mathrm{C}=$ ?

From $(\mathrm{I}), \therefore \quad \mathrm{C}=0.175 \times[1.75]^{\frac{20}{10}}$

$$
\begin{array}{ll}
\therefore & \mathrm{C}=0.175 \times[1.75]^{\frac{2}{1}} \\
\therefore & \mathrm{C}=0.175 \times 3.0625 \\
\therefore & \mathrm{C}=\mathbf{0 . 5 3 5 9 3 7 5} \text { crore }
\end{array}
$$

When $\mathrm{t}=30$ years from base; that is 15 August, 1977, what is $\mathrm{C}=$ ?

From $(\mathrm{I}), \therefore \quad \mathrm{C}=0.175 \times[1.75]^{\frac{30}{10}}$

$\therefore \quad \mathrm{C}=0.175 \times[1.75]^{\frac{3}{1}}$

$\therefore \quad \mathrm{C}=0.175 \times 5.359375$

$\therefore \quad \mathrm{C}=\mathbf{0 . 9 3 7 8 9 0 6 2 5}$ crore

When $\mathrm{t}=40$ years from base; that is 15 August, 1987, what is $\mathrm{C}=$ ?

From $(\mathrm{I}), \therefore \quad \mathrm{C}=0.175 \times[1.75]^{\frac{40}{10}}$

$\therefore \quad \mathrm{C}=0.175 \times[1.75]^{\frac{4}{1}}$

$\therefore \quad \mathrm{C}=0.175 \times 9.37890625$

$\therefore \quad \mathrm{C}=1.64130859375$ crore

When $\mathrm{t}=50$ years from base; that is 15 August, 1997 , what is $\mathrm{C}=$ ?

From $(\mathrm{I}), \therefore \quad \mathrm{C}=0.175 \times[1.75]^{\frac{50}{10}}$

$\therefore \quad \mathrm{C}=0.175 \times[1.75]^{\frac{5}{1}}$

$\therefore \quad \mathrm{C}=0.175 \times 16.4130859375$

$\therefore \quad \mathrm{C}=\mathbf{2 . 8 7 2 2 9 0}$ crore

When $\mathrm{t}=54$ years from base; that is 15 August, 2001, what is $\mathrm{C}=$ ?

From $(\mathrm{I}), \therefore \quad \mathrm{C}=0.175 \times[1.75]^{\frac{54}{10}}$

$$
\begin{array}{lll}
\therefore & \mathrm{C}=0.175 \times[1.75]^{\frac{5.4}{1}} \\
\therefore & \mathrm{C}=0.175 \times 20.53078064 \\
\therefore & \mathrm{C}=\mathbf{3 . 5 9 2 8 8 6 6} \text { crore }
\end{array}
$$

When $\mathrm{t}=55$ years from base; that is 15 August, 2002, what is $\mathrm{C}=$ ?

From $(\mathrm{I}), \therefore \quad \mathrm{C}=0.175 \times[1.75]^{\frac{55}{10}}$

$\therefore \quad \mathrm{C}=0.175 \times[1.75]^{\frac{5.5}{1}}$

$\therefore \quad \mathrm{C}=0.175 \times 21.7124718$

$\therefore \quad C=3.799682568$ crore

When $\mathrm{t}=56$ years from base; that is 15 August, 2003, what is $\mathrm{C}=$ ?

$$
\begin{array}{rlrl}
\text { From }(\mathrm{I}), & \therefore & \mathrm{C} & =0.175 \times[1.75]^{\frac{56}{10}} \\
\therefore & \mathrm{C} & =0.175 \times[1.75]^{\frac{5.6}{1}} \\
\therefore & \mathrm{C}=0.175 \times 22.96217765 \\
\therefore & \mathrm{C}=\mathbf{4 . 0 1 8 3 8 1 0 8 9} \text { crore }
\end{array}
$$

When $\mathrm{t}=57$ years from base; that is 15 August, 2004, what is $\mathrm{C}=$ ?

From $(\mathrm{I}), \therefore \quad \mathrm{C}=0.175 \times[1.75]^{\frac{57}{10}}$

$$
\begin{array}{ll}
\therefore & \mathrm{C}=0.175 \times[1.75]^{\frac{5.7}{1}} \\
\therefore & \mathrm{C}=0.175 \times 24.283812867
\end{array}
$$

$$
\therefore \quad \mathrm{C}=\mathbf{4 . 2 4 9 6 6 7 2 5 \text { crore }}
$$

When $\mathrm{t}=58$ years from base; that is 15 August, 2005, what is $\mathrm{C}=$ ?

From $(\mathrm{I}), \therefore \quad \mathrm{C}=0.175 \times[1.75]^{\frac{58}{10}}$

$$
\begin{array}{ll}
\therefore & \mathrm{C}=0.175 \times[1.75]^{\frac{5.8}{1}} \\
\therefore & \mathrm{C}=0.175 \times 25.6815175069 \\
\therefore & \mathrm{C}=\mathbf{4 . 4 9 4 2 6 5 5 6} \text { crore }
\end{array}
$$

When $\mathrm{t}=59$ years from base; that is 15 August, 2006, what is $\mathrm{C}=$ ?

$$
\begin{aligned}
\text { From }(\mathrm{I}), \therefore & \mathrm{C}=0.175 \times[1.75]^{\frac{59}{10}} \\
\therefore & \mathrm{C}=0.175 \times[1.75]^{\frac{5.9}{1}} \\
\therefore & \mathrm{C}=0.175 \times 27.1596699
\end{aligned}
$$




\section{$\therefore \quad C=4.7529422329998$ crore}

When $\mathrm{t}=60$ years from base; that is 15 August, 2007, what is $\mathrm{C}=$ ?

From $(\mathrm{I}), \therefore \quad \mathrm{C}=0.175 \times[1.75]^{\frac{60}{10}}$

$\therefore \quad \mathrm{C}=0.175 \times[1.75]^{\frac{6}{1}}$

$\therefore \quad \mathrm{C}=0.175 \times 28.722900390$

$\therefore \quad \mathrm{C}=\mathbf{5 . 0 2 6 5 0 7 5 6 8}$ crore

When $\mathrm{t}=61$ years from base; that is 15 August, 2008, what is $\mathrm{C}=$ ?

From $(\mathrm{I}), \therefore \quad \mathrm{C}=0.175 \times[1.75]^{\frac{61}{10}}$

$\therefore \quad \mathrm{C}=0.175 \times[1.75]^{\frac{6.1}{1}}$

$\therefore \quad \mathrm{C}=0.175 \times 30.3761058$

$\therefore \quad \mathbf{C}=\mathbf{5 . 3 1 5 8 1 8 5 1 7 \text { crore }}$

When $\mathrm{t}=62$ years from base; that is 15 August, 2009, what is $\mathrm{C}=$ ?

From $(\mathrm{I}), \therefore \quad \mathrm{C}=0.175 \times[1.75]^{\frac{62}{10}}$

$\therefore \quad \mathrm{C}=0.175 \times[1.75]^{\frac{6.2}{1}}$

$\therefore \quad \mathrm{C}=0.175 \times 32.1244648657$

$\therefore \quad \mathbf{C}=\mathbf{5 . 6 2 1 7 8 1 3 5 c r o r e}$

When $\mathrm{t}=63$ years from base; that is 15 August, 2010, what is $\mathrm{C}=$ ?

From $(\mathrm{I}), \therefore \quad \mathrm{C}=0.175 \times[1.75]^{\frac{63}{10}}$

$$
\begin{array}{ll}
\therefore & \mathrm{C}=0.175 \times[1.75]^{\frac{6.3}{1}} \\
\therefore & \mathrm{C}=0.175 \times 33.97345430 \\
\therefore & \mathrm{C}=\mathbf{5 . 9 4 5 3 5 4 5 0 3 7 \text { crore }}
\end{array}
$$

When $\mathrm{t}=64$ years from base; that is 15 August, 2011, what is $\mathrm{C}=$ ?

From $(I), \therefore \quad C=0.175 \times[1.75]^{\frac{64}{10}}$

$$
\begin{array}{ll}
\therefore & \mathrm{C}=0.175 \times[1.75]^{\frac{6.4}{1}} \\
\therefore & \mathrm{C}=0.175 \times 35.928866612
\end{array}
$$$$
\therefore \quad \mathrm{C}=\mathbf{6 . 2 8 7 5 5 1 5 7 2 1 \mathrm { crore }}
$$

When $\mathrm{t}=65$ years from base; that is 15 August, 2012, what is $\mathrm{C}=$ ?

From $(\mathrm{I}), \therefore \quad \mathrm{C}=0.175 \times[1.75]^{\frac{65}{10}}$

$\therefore \quad \mathrm{C}=0.175 \times[1.75]^{\frac{6.5}{1}}$

$\therefore \quad \mathrm{C}=0.175 \times 37.9968256830$

$\therefore \quad C=6.64944449453$ crore

When $\mathrm{t}=66$ years from base; that is 15 August, 2013, what is $\mathrm{C}=$ ?

$$
\text { From (I ), } \begin{aligned}
\therefore & \mathrm{C}=0.175 \times[1.75]^{\frac{66}{10}} \\
\therefore & \mathrm{C}=0.175 \times[1.75]^{\frac{6.6}{1}} \\
\therefore & \mathrm{C}=0.175 \times 40.183810891 \\
\therefore & \mathrm{C}=7.0321669060 \mathrm{crore}
\end{aligned}
$$

When $\mathrm{t}=67$ years from base; that is 15 August, 2014, what is $\mathrm{C}=$ ?

From $(\mathrm{I}), \therefore \quad \mathrm{C}=0.175 \times[1.75]^{\frac{67}{10}}$

$$
\begin{array}{ll}
\therefore & \mathrm{C}=0.175 \times[1.75]^{\frac{6.7}{1}} \\
\therefore & \mathrm{C}=0.175 \times 42.49667251 \\
\therefore & \mathrm{C}=7.436917690 \text { crore }
\end{array}
$$

From the above we have observed and try to find that the total Income tax holder for a particular period in the country India.

\subsubsection{Mathematical Result:}

Table: I

\begin{tabular}{|c|c|c|c|c|}
\hline $\begin{array}{l}\text { Mathematical Model Period ' } \mathrm{t} \text { ' } \\
\text { year }\end{array}$ & $\begin{array}{l}\text { Total Income tax Holder 'C' } \\
\text { crore }\end{array}$ & $\begin{array}{l}\text { Income tax not Paid } \\
\text { Holder 'C' crore }\end{array}$ & $\begin{array}{l}\text { Income tax Paid Holder } \\
\text { 'C' crore }\end{array}$ \\
\hline 0 & 0.175 & 0 & 0.175 \\
\hline 10 & 0.30625 & 0 & 0.30625 \\
\hline 20 & 0.5359375 & 0.0546875 & 0.48125 \\
\hline 30 & 0.937890625 & 0.281640625 & 0.65625 \\
\hline
\end{tabular}


Mathematical Modelling: A Study of Corruption in Various fields of the Society

\begin{tabular}{|c|c|c|c|}
\hline 40 & 1.641308594 & 0.810058594 & 0.83125 \\
\hline 50 & 2.872290 & 1.00625 & 1.86604 \\
\hline 54 & 3.5928866 & 1.18125 & 2.4116366 \\
\hline 55 & 3.799682568 & 1.35625 & 2.443432568 \\
\hline 56 & 4.018381089 & 1.53125 & 2.487131089 \\
\hline 57 & 4.24966725 & 1.70625 & 2.54341725 \\
\hline 58 & 4.49426556 & 1.88125 & 2.61301556 \\
\hline 59 & 4.752942233 & 2.05625 & 2.696692233 \\
\hline 60 & 5.026507568 & 2.23125 & 2.795257568 \\
\hline 61 & 5.315818517 & 2.40625 & 2.909568517 \\
\hline 62 & 5.62178135 & 2.58125 & 3.04053135 \\
\hline 63 & 5.945354504 & 2.75625 & 3.189104504 \\
\hline 64 & 6.2875515721 & 2.93125 & 3.3563015721 \\
\hline 65 & 6.6494444945 & 3.10625 & 3.5431944945 \\
\hline 66 & 7.0321669060 & 3.28125 & 3.750916906 \\
\hline 67 & 7.436917690 & 3.45625 & 3.98066769 \\
\hline
\end{tabular}

\subsubsection{Mathematical Graph:}

The graph of the mathematical result is shown in the following:

\section{Graph: I}

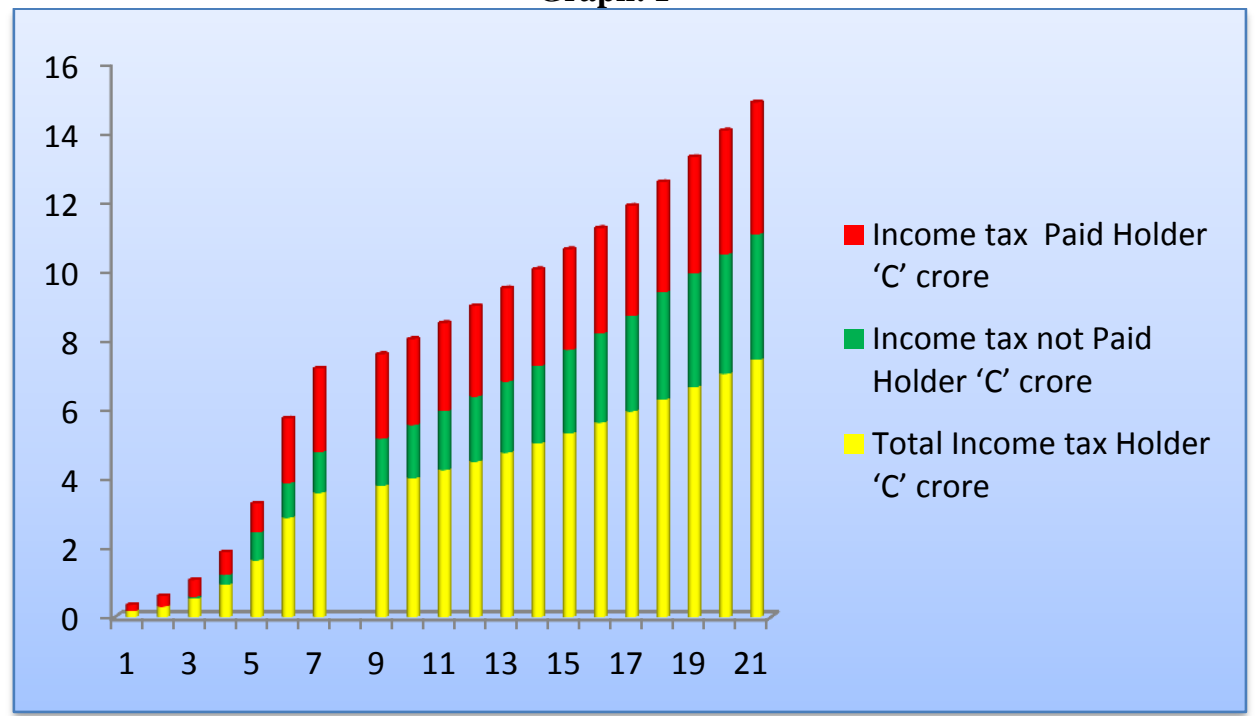

From the graph we have seen that the total number of Income tax holder in which some Income tax holder have paid or not paid or miss Income tax holder have shown for a particular period in the country India.

\subsubsection{Mathematical Development in the field of Income tax bureaucracy except Corruption:}

I will try to find the amount of Income tax Holder for related period. Therefore we assume that when $\mathrm{C}=0$ then $\mathrm{D}(0)=0.35$ crore (that is $1 \%$ of total population) at 15 August, 1947. Therefore we know that Mathematical Corruption-Development model with difference equation form, it is as

$$
\begin{array}{ll}
\therefore & \mathrm{D}(\mathrm{C})=\mathrm{D}(0)[1+\mathrm{K}]^{\mathrm{C}} \\
\therefore & 0.35=\mathrm{D}(0)[1+\mathrm{K}]^{0} \\
\therefore & \mathrm{D}(0)=0.35 \text { crore }
\end{array}
$$

Putting in equation (i), we get

$$
\therefore \quad \mathrm{D}(\mathrm{C})=0.35 \times[1+\mathrm{K}]^{\mathrm{C}}
$$

Further after $\mathrm{t}=10$ years Mathematical Model period, $\mathrm{D}(\mathrm{C})=0.6125$, when $\mathrm{C}=0.31$ that is $7 / 4$ of $\mathrm{D}(0)$.

Therefore from equation (ii), we have

$$
\begin{array}{ll}
\therefore & 0.6125=0.35 \times[1+K]^{0.31} \\
\therefore & 1+K=\left[\frac{0.6125}{0.35}\right]^{\frac{1}{0.31}}
\end{array}
$$

Putting this value in equation (ii), we have 


$$
\begin{array}{ll}
\therefore & \mathrm{D}(\mathrm{C})=0.35 \times\left[\frac{0.6125}{0.35}\right]^{\frac{C}{0.31}} \\
\therefore & \mathbf{D}(\mathbf{C})=\mathbf{0 . 3 5} \times[\mathbf{1 . 7 5}]^{\frac{C}{0.31}}
\end{array}
$$

This is known as Mathematical Model base Development with related Corruption formula.

Now when $\mathrm{t}=0, \mathrm{C}=0.175$ ( $0.5 \%$ of population) on 15 August, 1947 , what is $\mathrm{D}(\mathrm{C})=$ ?

Therefore from equation (iii), we have

$$
\begin{array}{ll}
\therefore & \mathrm{D}(\mathrm{C})=0.35 \times[1.75]^{\frac{0.175}{0.31}} \\
\therefore & \mathrm{D}(\mathrm{C})=0.35 \times[1.75]^{0.56} \\
\therefore & \mathrm{D}(\mathbf{C})=\mathbf{0 . 4 7 8 8 1 6 7 6 8 8} \text { crore }
\end{array}
$$

When $\mathrm{t}=10$ years, $\mathrm{C}=0.30625$ crore on 15 August, 1957 , then what is $\mathrm{D}(\mathrm{C})=$ ?

Therefore from equation (iii), we have

$$
\begin{array}{ll}
\therefore & \mathrm{D}(\mathrm{C})=0.35 \times[1.75]^{\frac{0.30625}{0.31}} \\
\therefore & \mathrm{D}(\mathrm{C})=0.35 \times[1.75]^{0.99} \\
\therefore & \mathrm{D}(\mathbf{C})=\mathbf{0 . 6 0 9 0 8 1 9 2 6} \text { crore }
\end{array}
$$

When $\mathrm{t}=20$ years, $\mathrm{C}=0.5359375$ crore on 15 August, 1967 , then what is $\mathrm{D}(\mathrm{C})=$ ?

Therefore from equation (iii), we have

$$
\begin{array}{lll}
\therefore & \mathrm{D}(\mathrm{C}) & =0.35 \times[1.75]^{\frac{0.5359375}{0.31}} \\
\therefore & \mathrm{D}(\mathrm{C}) & =0.35 \times[1.75]^{1.7288} \\
\therefore & \mathrm{D}(\mathbf{C}) & =\mathbf{0 . 9 2 0 9 4 1 8 7 9 7} \text { crore }
\end{array}
$$

When $\mathrm{t}=30$ years, $\mathrm{C}=0.937890625$ crore on 15 August, 1977 , then what is $\mathrm{D}(\mathrm{C})=$ ? Therefore from equation (iii), we have

$$
\begin{array}{ll}
\therefore & \mathrm{D}(\mathrm{C})=0.35 \times[1.75]^{\frac{0.937890625}{0.31}} \\
\therefore & \mathrm{D}(\mathrm{C})=0.35 \times[1.75]^{3.02545} \\
\therefore & \mathrm{D}(\mathrm{C})=0.35 \times 5.436250547 \\
\therefore & \mathrm{D}(\mathbf{C})=\mathbf{1 . 9 0 2 6 8 7 6 9 1} \text { crore }
\end{array}
$$

When $\mathrm{t}=40$ years, $\mathrm{C}=1.641308594$ crore on 15 August, 1987, then what is $\mathrm{D}(\mathrm{C})=$ ? Therefore from equation (iii), we have

$$
\begin{array}{ll}
\therefore & \mathrm{D}(\mathrm{C})=0.35 \times[1.75]^{\frac{1.641308594}{0.31}} \\
\therefore & \mathrm{D}(\mathrm{C})=0.35 \times[1.75]^{5.29454} \\
\therefore & \mathrm{D}(\mathrm{C})=0.35 \times 19.354175 \text { crore } \\
\therefore & \mathrm{D}(\mathrm{C})=\mathbf{6 . 7 7 3 9 6 1 3 5 4 1} \text { crore }
\end{array}
$$

When $\mathrm{t}=50$ years, $\mathrm{C}=2.872290$ crore on 15 August, 1997 , then what is $\mathrm{D}(\mathrm{C})=$ ?

Therefore from equation (iii), we have

$$
\begin{array}{ll}
\therefore & \mathrm{D}(\mathrm{C})=0.35 \times[1.75]^{\frac{2.872290}{0.31}} \\
\therefore & \mathrm{D}(\mathrm{C})=0.35 \times[1.75]^{9.2655} \\
\therefore & \mathrm{D}(\mathrm{C})=0.35 \times 178.5948947 \text { crore } \\
\therefore & \mathrm{D}(\mathbf{C})=\mathbf{6 2 . 5 0 8 2 1 3 2} \text { crore }
\end{array}
$$

When $\mathrm{t}=54$ years, $\mathrm{C}=3.5928866$ crore on 15 August, 2001 , then what is $\mathrm{D}(\mathrm{C})=$ ?

Therefore from equation (iii), we have

$$
\begin{array}{lll}
\therefore & \mathrm{D}(\mathrm{C}) & =0.35 \times[1.75]^{\frac{3.5928866}{0.31}} \\
\therefore & \mathrm{D}(\mathrm{C}) & =0.35 \times[1.75]^{11.5899} \\
\therefore & \mathrm{D}(\mathrm{C}) & =0.35 \times 655.8230558 \text { crore } \\
\therefore & \mathrm{D}(\mathrm{C}) & =\mathbf{2 2 9 . 5 3 8 0 6 9 5} \text { crore }
\end{array}
$$

When $\mathrm{t}=55$ years, $\mathrm{C}=3.799682568$ crore on 15 August, 2002, then what is $\mathrm{D}(\mathrm{C})=$ ?

Therefore from equation (iii), we have

$$
\begin{array}{ll}
\therefore & \mathrm{D}(\mathrm{C})=0.35 \times[1.75]^{\frac{3.799682568}{0.31}} \\
\therefore & \mathrm{D}(\mathrm{C})=0.35 \times[1.75]^{12.25704} \\
\therefore & \mathrm{D}(\mathrm{C})=0.35 \times 952.6362081 \text { crore } \\
\therefore & \mathrm{D}(\mathbf{C})=\mathbf{3 3 3 . 4 2 2 6 7 2 8} \text { crore }
\end{array}
$$

When $\mathrm{t}=56$ years, $\mathrm{C}=4.018381089$ crore on 15 August, 2003, then what is $\mathrm{D}(\mathrm{C})=$ ? Therefore from equation (iii), we have

$$
\begin{array}{ll}
\therefore & \mathrm{D}(\mathrm{C})=0.35 \times[1.75]^{\frac{4.018381089}{0.31}} \\
\therefore & \mathrm{D}(\mathrm{C})=0.35 \times[1.75]^{12.96252} \\
\therefore & \mathrm{D}(\mathrm{C})=0.35 \times 1413.792152996 \text { crore } \\
\therefore & \mathrm{D}(\mathbf{C})=\mathbf{4 9 4 . 8 2 7 2 5 3 5 5} \text { crore }
\end{array}
$$


When $\mathrm{t}=57$ years, $\mathrm{C}=4.24966725$ crore on 15 August, 2004 , then what is $\mathrm{D}(\mathrm{C})=$ ?

Therefore from equation (iii), we have

$$
\begin{array}{ll}
\therefore & \mathrm{D}(\mathrm{C})=0.35 \times[1.75]^{13.70860} \\
\therefore & \mathrm{D}(\mathrm{C})=0.35 \times 2146.403630 \text { crore } \\
\therefore & \mathrm{D}(\mathbf{C})=\mathbf{7 5 1 . 2 4 1 2 7 0 6 3 3} \text { crore }
\end{array}
$$

When $\mathrm{t}=58$ years, $\mathrm{C}=4.49426556$ crore on 15 August, 2005 , then what is $\mathrm{D}(\mathrm{C})=$ ?

Therefore from equation (iii), we have

$$
\begin{array}{ll}
\therefore & \mathrm{D}(\mathrm{C})=0.35 \times[1.75]^{\frac{4.49426556}{0.31}} \\
\therefore & \mathrm{D}(\mathrm{C})=0.35 \times[1.75]^{14.4976} \\
\therefore & \mathrm{D}(\mathrm{C})=0.35 \times 3337.862287 \text { crore } \\
\therefore & \mathrm{D}(\mathbf{C})=\mathbf{1 1 6 8 . 2 5 1 8 0 0} \text { crore }
\end{array}
$$

When $\mathrm{t}=59$ years, $\mathrm{C}=4.752942233$ crore on 15 August, 2006, then what is $\mathrm{D}(\mathrm{C})=$ ?

Therefore from equation (iii), we have

$$
\begin{array}{ll}
\therefore & \mathrm{D}(\mathrm{C})=0.35 \times[1.75]^{\frac{4.752942233}{0.31}} \\
\therefore & \mathrm{D}(\mathrm{C})=0.35 \times[1.75]^{15.3321} \\
\therefore & \mathrm{D}(\mathrm{C})=0.35 \times 5324.55928 \text { crore } \\
\therefore & \mathrm{D}(\mathbf{C})=\mathbf{1 8 6 3 . 5 9 5 7 4 6 8} \text { crore }
\end{array}
$$

When $\mathrm{t}=60$ years, $\mathrm{C}=5.026507568$ crore on 15 August, 2007 , then what is $\mathrm{D}(\mathrm{C})=$ ? Therefore from equation (iii), we have

$$
\begin{array}{ll}
\therefore & \mathrm{D}(\mathrm{C})=0.35 \times[1.75]^{\frac{5.026507568}{0.31}} \\
\therefore & \mathrm{D}(\mathrm{C})=0.35 \times[1.75]^{16.2145} \\
\therefore & \mathrm{D}(\mathrm{C})=0.35 \times 8724.497859 \text { crore } \\
\therefore & \mathrm{D}(\mathbf{C})=\mathbf{3 0 5 3 . 5 7 4 2 5 0 7 7 6 8 \text { crore }}
\end{array}
$$

When $\mathrm{t}=61$ years, $\mathrm{C}=5.315818517$ crore on 15 August, 2008 , then what is $\mathrm{D}(\mathrm{C})=$ ?

Therefore from equation (iii), we have

$$
\begin{array}{ll}
\therefore & \mathrm{D}(\mathrm{C})=0.35 \times[1.75]^{\frac{5.315818517}{0.31}} \\
\therefore & \mathrm{D}(\mathrm{C})=0.35 \times[1.75]^{17.14780} \\
\therefore & \mathrm{D}(\mathrm{C})=0.35 \times 14708.48193 \text { crore } \\
\therefore & \mathrm{D}(\mathbf{C})=\mathbf{5 1 4 7 . 9 6 8 6 7 5 3} \text { crore }
\end{array}
$$

When $\mathrm{t}=62$ years, $\mathrm{C}=5.62178135$ crore on 15 August, 2009 , then what is $\mathrm{D}(\mathrm{C})=$ ?

Therefore from equation (iii), we have

$$
\begin{array}{ll}
\therefore & \mathrm{D}(\mathrm{C})=0.35 \times[1.75]^{\frac{5.62178135}{0.31}} \\
\therefore & \mathrm{D}(\mathrm{C})=0.35 \times[1.75]^{18.13478} \\
\therefore & \mathrm{D}(\mathrm{C})=0.35 \times 25552.979383 \text { crore } \\
\therefore & \mathrm{D}(\mathbf{C})=\mathbf{8 9 4 3 . 5 4 2 7 8 4 0} \text { crore }
\end{array}
$$

When $\mathrm{t}=63$ years, $\mathrm{C}=5.945354504$ crore on 15 August, 2010, then what is $\mathrm{D}(\mathrm{C})=$ ? Therefore from equation (iii), we have

$$
\begin{array}{ll}
\therefore & \mathrm{D}(\mathrm{C})=0.35 \times[1.75]^{\frac{5.945354504}{0.31}} \\
\therefore & \mathrm{D}(\mathrm{C})=0.35 \times[1.75]^{19.17857} \\
\therefore & \mathrm{D}(\mathrm{C})=0.35 \times 45827.084595 \text { crore } \\
\therefore & \mathrm{D}(\mathbf{C})=\mathbf{1 6 0 3 9 . 4 7 9 6 0 8 3 7} \text { crore }
\end{array}
$$

When $\mathrm{t}=64$ years, $\mathrm{C}=6.2875515721$ crore on 15 August, 2011 , then what is $\mathrm{D}(\mathrm{C})=$ ?

Therefore from equation (iii), we have

$$
\begin{array}{ll}
\therefore & \mathrm{D}(\mathrm{C})=0.35 \times[1.75]^{\frac{6.2875515721}{0.31}} \\
\therefore & \mathrm{D}(\mathrm{C})=0.35 \times[1.75]^{20.28242} \\
\therefore & \mathrm{D}(\mathrm{C})=0.35 \times 84996.25278 \text { crore } \\
\therefore & \mathrm{D}(\mathbf{C})=\mathbf{2 9 7 4 8 . 6 8 8 4 7 2 3} \text { crore }
\end{array}
$$

When $\mathrm{t}=65$ years, $\mathrm{C}=6.6494444945$ crore on 15 August, 2012 , then what is $\mathrm{D}(\mathrm{C})=$ ? Therefore from equation (iii), we have

$$
\begin{array}{ll}
\therefore & \mathrm{D}(\mathrm{C})=0.35 \times[1.75]^{\frac{6.6494444945}{0.31}} \\
\therefore & \mathrm{D}(\mathrm{C})=0.35 \times[1.75]^{21.4498} \\
\therefore & \mathrm{D}(\mathrm{C})=0.35 \times 163349.39741 \text { crore } \\
\therefore & \mathrm{D}(\mathbf{C})=\mathbf{5 7 1 7 2 . 2 8 9 0 9 2 6} \text { crore }
\end{array}
$$

When $t=66$ years, $C=7.0321669060$ crore on 15 August, 2013, then what is $D(C)=$ ? Therefore from equation (iii), we have 


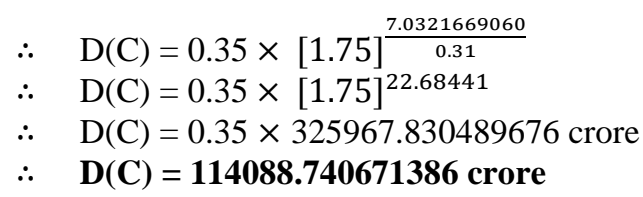

When $\mathrm{t}=67$ years, $\mathrm{C}=7.436917690$ crore on 15 August, 2014, then what is $\mathrm{D}(\mathrm{C})=$ ?

Therefore from equation (iii), we have

$$
\begin{array}{ll}
\therefore & \mathrm{D}(\mathrm{C})=0.35 \times[1.75]^{\frac{7.436917690}{0.31}} \\
\therefore & \mathrm{D}(\mathrm{C})=0.35 \times[1.75]^{23.9901} \\
\therefore & \mathrm{D}(\mathrm{C})=0.35 \times 676872.8457893 \text { crore } \\
\therefore & \mathrm{D}(\mathbf{C})=\mathbf{2 3 6 9 0 5 . 4 9 6 0 2 6 2 6} \text { crore }
\end{array}
$$

3.1.3.1 Mathematical Result:

From the above we have found the mathematical result of earlier example. There are as of the following table:

Table: II

\begin{tabular}{|c|c|c|c|c|}
\hline $\begin{array}{c}\text { Mathematical } \\
\text { Model Period ' } \mathrm{t} \text { ' } \\
\text { year }\end{array}$ & $\begin{array}{c}\text { Total Income tax } \\
\text { Holder 'C' crore }\end{array}$ & $\begin{array}{c}\text { Income tax total amount 'C' } \\
\text { crore }\end{array}$ & $\begin{array}{c}\text { Income tax Paid } \\
\text { amount 'C' crore }\end{array}$ & $\begin{array}{c}\text { Income tax not paid amount } \\
\text { 'C' crore }\end{array}$ \\
\hline 0 & 0.175 & 0.4788167688 & 0.4788167688 & 0 \\
\hline 10 & 0.30625 & 0.609081926 & 0.609081926 & 0.0939736612 \\
\hline 20 & 0.5359375 & 0.9209418797 & 0.8269682185 & 0.5713610268 \\
\hline 30 & 0.937890625 & 1.902687691 & 1.3313266642 & 3.3432503981 \\
\hline 40 & 1.641308594 & 6.7739613541 & 3.430710956 & 21.8985163519 \\
\hline 50 & 2.872290 & 62.5082132 & 40.6096968481 & 75.4663788 \\
\hline 54 & 3.5928866 & 229.5380695 & 154.0716907 & 119.0111258 \\
\hline 55 & 3.799682568 & 333.4226728 & 214.4115470 & 301.55957545 \\
\hline 56 & 4.018381089 & 494.82725355 & 306.2676781 & 489.01732 \\
\hline 57 & 4.24966725 & 751.241270633 & 449.61638035 & 806.2413904 \\
\hline 58 & 4.49426556 & 1168.251800 & 679.234480 & 1355.4714590368 \\
\hline 59 & 4.752942233 & 1863.5957468 & 1057.3543564 & 2330.271356204 \\
\hline 60 & 5.026507568 & 3053.5742507768 & 1698.10279174 & 4106.442135321 \\
\hline 61 & 5.31581852 & 5147.9686753 & 2817.697319096 & 7435.85864238 \\
\hline 62 & 5.62178135 & 8943.5427840 & 4837.100648679 & 14696.7961229 \\
\hline 63 & 5.945354504 & 16039.47960837 & 8603.62096599 & 28222.367776 \\
\hline 64 & 6.2875515721 & 29748.6884723 & 15051.8923494 & 56073.642053186 \\
\hline 65 & 6.6494444495 & 57172.2890926 & 28959.9213166 & 115674.68113868 \\
\hline 66 & 7.0321669060 & 114088.740671386 & 58015.0986182 & \\
\hline 67 & 7.436917690 & 236905.49602626 & 121230.81488758 & \\
\hline 3.1 .2 & & & & \\
\hline
\end{tabular}

\subsubsection{Mathematical Graph:}

From the above calculated data in the table shows that how many Income tax holders in the country India and their total amount in the form of Income tax total amount, Income tax paid amount and Income tax not or less paid amount. There are shown in the following graph:

\section{Graph: II}

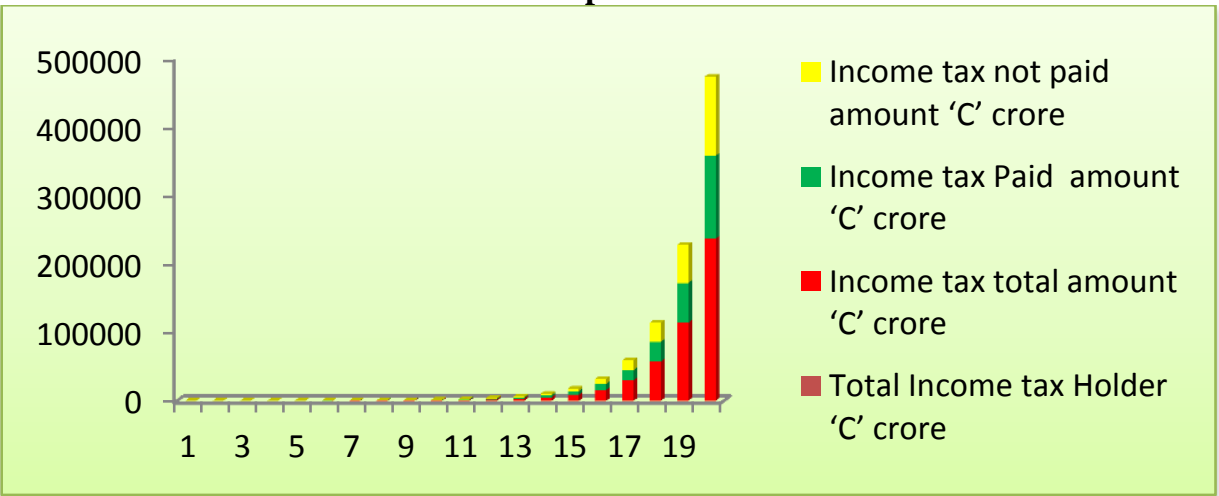

We have observed from Table-I, Table-II, Graph-I and Graph-II that the total Income tax holder and their amount have increased for every year from 15 August 1947. Also we have observed that among these IT-holder, 
some have paid income tax and some have not (less or miss) paid income tax and both are increased for every year from 15 August 1947.

For every time we have used mathematical modelling that means we have given a real world problem "Corruption" that is "for removing corruption from the society" convert this problem in to the mathematics problem. Further by using seventh step of mathematical modelling, we have found mathematical corruption model with difference equation form. Then for every mathematical model period we can found mathematical result and when we compare this mathematical result with the real world mathematical result if it is true then we get mathematical solution of the real world problem.

\section{Conclusion:}

In this paper we have studied the topic for measuring and removing corruption from the society by using mathematical modelling. Also we have observed and it concluded that the real world problem "corruption" from the society when we convert this problem in to mathematics problem then we get some models such as 'Mathematical Corruption Model, Mathematical Corruption Control Model, Mathematical Corruption-Development Model, Mathematical Development Model and Mathematical E-virus Constant Model'. They are used for measuring as well as removing the corruption from the society of any country of the world. Also we know that India is the largest democracy in the world. So it continues to struggle on a daily basis to fight corruption in the various fields of the society such as in politics at both the national and local levels different government as well as private offices. In India, there are a rich diversity of languages, cultures and traditions, nothing is more important to reconciling all the differences than the right for vote. However, the democratically elected government seemingly does nothing to bridge the enormous gap between the rich and the poor and to make the lives of the 500 or 600 million people living below the poverty line any better. Also we know in India there are more people living in poverty than any other nation and therefore for finding a solution to these basic issues of human rights has recently taken a backseat to nuclear weapons testing and other extravagant nationalist issues on the Indian political agenda.

Furthermore, the instability and corruption of the government since India won its independence in 15 August, 1947 has discouraged the long-term investments that are needed to drive economic growth. The fact of the matter is that India is on far with the United States as one of the models of democracy in the world; yet, India is far behind the United States in its economic development. There can only be one explanation for India's unimpressive economic record and maximum peoples living in poverty the unprecedented political corruption and instability that can so easily be seen at all levels of government. Unfortunately, there are very few means for the citizens to fight the corruption in the current system. In this connection we have seen from the above two illustrations that the total number of people have income tax holder and among these people, approximately 50\% people have paid income tax sincerely. There are shown of the following:

Table: III

\begin{tabular}{|l|l|l|}
\hline $\begin{array}{l}\text { Mathematical } \\
\text { period t year }\end{array}$ & $\begin{array}{l}\text { Mathematical Result in Real world Income } \\
\text { tax Paid holder (crore) }\end{array}$ & $\begin{array}{l}\text { Mathematical Result in (calculated) } \\
\text { Mathematics world Income tax Paid holder } \\
\text { crore) }\end{array}$ \\
\hline $2005-2006$ & 2.98 & 2.7459749 \\
\hline $2006-2007$ & 3.13 & 2.8524130425 \\
\hline $2007-2008$ & 3.36 & 2.9750499335 \\
\hline $2008-2009$ & 3.27 & 3.114817927 \\
\hline $2009-2010$ & 3.41 & 3.27270303805 \\
\hline $2010-2011$ & 3.48 & 3.4497480333 \\
\hline $2011-2012$ & 3.65 & 3.64705570025 \\
\hline $2012-2013$ & 3.87 & 3.865792298 \\
\hline $2013-2014$ & 3.99 & 3.98066769 \\
\hline
\end{tabular}

The above table shows that the comparatively mathematical study between the real world problem and mathematics world. Also we check the validity from the above two results that is both the results are approximately equal. That is our Mathematical Corruption Model is valid for the above illustrations.

\section{References:}

[1]. Andriole SJ (1983) Handbook of Problem Solving: An Analytic Methodology Princeton, NJ: Petrocelli Books.

[2]. Blum, W., Niss, M. (1991). Applied Mathematical Problem Solving, Modeling, Applications and links to other subjects- state, trends and issues in mathematics instruction. In: Educational studies in Mathematics, 22(1), 37-68.

[3]. BhaskarDasgupta, Applied Mathematical Methods published by Darling Kindersley (India) Pvt. Ltd. Delhi.

[4]. Bender EA (1978) An Introduction to Mathematical Modeling New York: John Wiley and Sons.

[5]. Daniel A. Murray, Introductory Course in Differential equations, Orient Longman ltd. Harlow and London (1993).

[6]. De Lange, J. (1996). Real Problems with Real world Mathematics. In L. Alsina\& al. (Eds.), proceeding of the 8th Int. Congress on Math.1 Education (pp. 83-110). Seville: Thales.

[7]. Nicholas Charron, Ph.D, The Correlates of Corruption in India: Analysis and Evidence from the states 
[8]. Rose-Ackerman, Susan 1999. 'Corruption and Government: Causes Consequences and Reform. Cambridge University Press, New York.

[9]. Schoenfeld A. H. (1994). Mathematical Thinking and Problem Solving. Hillsdale: Erlbaum

[10] Stillman G (2002) Assessing Higher order Mathematical Thinking through Applications Ph.D Thesis) Brisbane: The university of Queensland.

[11]. Shleifer, Andrei and Vishny, R. W. 1993. 'Corruption' Quarterly Journal of Economics 108:599-17

[12]. Siddhartha Tak, Zee Research GroupTaxpayers fail to keep pace with PAN card holders Last Updated: September 04, $2011,17: 35$.

[13]. Stillman G (2002) Assessing Higher order Mathematical Thinking through Applications Ph.D Thesis) Brisbane: The university of Queensland.

[14]. S. J. Lamon, W. A. Parker \& K. Houston (Eds.); Mathematical Modelling: A way of life Chichester: Harwood Publishing.Chichester: Ellis Harwood; Modelling, Applications and applied problem solving.

[15]. Treilibs, V; Burkhardt, H; and Low, 1 (1980). Formulation processes in Mathematical Modelling; Nottingham: shell centre for Mathematical Education.

[16]. Transparency International (2005). 'India Corruption Study 2005' Published by Transparency International India LajpatBhawan, Lajpat Nagar IV, New Delhi

[17]. Treisman, D. (2000). 'The Causes of Corruption: A Cross-National Study'. Journal of Public Economics, 76(3): 399-458

[18]. Verschaffel, L.; Greer, B.; DeCorte, E. (2000). Making sense of world Problems.Lisse: Swets\&Zeitlinger.

[19]. VarunGajendran, Professor Lusignan, TA: SahilKhanna; EDGE Research Paper:The Implications of Political Corruption for India's Economic Development.

[20]. World Bank 1997. Helping Countries Combat Corruption: The Role of the World Bank.SeptemberReport of a task force on Corruption Action Plan Working Group, Coordinated by Mike Stevens.

[21]. Mauro, Paolo (1995). 'Corruption and Growth'.Quarterly Journal of Economics 110:681- 712. 